ACM Proceeding of the International Conference

on Computer Graphics, Imaging and Visualization, CGIV'0,

Penang, Malaysia, July 2004, pp. 159-164

\title{
A Framework for Polysensometric Multidimensional Spatial Visualization
}

\author{
Javed Khan, Xuebin Xu, and Yongbin Ma \\ Media Communications and Networking Research Laboratory, \\ Department of Math \& Computer Science, Kent State University, \\ 233 MSB, Kent, $\mathrm{OH} 44242$ \\ \{javed,xxu1,yma\}@kent.edu
}

\begin{abstract}
Typically any single sensor instrument suffers from physical/observation constraints. This paper discusses a generalized framework, called polymorphic visual information fusion framework (PVIF) that can enable information from multiple sensors to be fused and compared to gain broader understanding of a target of observation in multidimensional space. An automate software system supporting comparative cognition has been developed to form 3D models based on the datasets from different sensors, such as XPS and LSCM. This fusion framework not only provides an information engineering based tool to overcome the limitations of individual sensor's scope of observation but also provides a means where theoretical understanding surrounding a complex target can be mutually validated by comparative cognition about the object of interest and 3D model refinement. Some polysensometric data classification metrics are provided to measure the quality of input datasets for fusion visualization.
\end{abstract}

\section{Introduction}

$3 \mathrm{D}$ visualization is becoming widespread and is being used in many fields. However, a relatively new area is comparative visualization. The methodology of correlating information from different experimental techniques to find the 3D structures improves scientists' understanding about the objects they work on. This has been recently demonstrated in a growth in interest in combining $C T$ (computerized tomography) and MRI (magnetic resonance imaging) information $[3,4]$. Comparative cognition is an important and strong means to obtain new knowledge about any complex new object or system. Multiple sets of data are collected and compared in the process. How to make use of these data sets is a complex issue and depends on what objects are worked on, which field the research is in and what purpose of the research is, etc. The most straightforward usage is to combine the data sets to obtain a composed model about the interest object when these multiple sets of data are complementary to one another. This is a data additive process for increasing the visibility of object of interest. Current research in the area mainly has produced very domain specific systems which fused data from very specific modes. In our project we investigate and propose how a generalized fusion framework can be constructed. We propose an algorithmic model of multi-sensor information fusion that closely correspond the cognitive process of comparison based knowledge exploration and hypothesis refinement. The overlapped parts among polysensometric datasets are registered and cropped out to form a refined 3D model, which is closer to the real structure of object of interest than that from single sensor. This is subtractive process for 3D model refinement. Along with it we have also developed a new automated system. It accepts sets of information from multiple techniques and combines them for $3 \mathrm{D}$ visualization. In the system, all data are mapped into one domain. Also, new data can be injected based on the knowledge of the field of the system user. The system can be easily extended to other new instrument. Naturally the user knows the physical-chemical meaning of data and has the proper library to process the data and accordingly the user can insert domain specific processed and reuse 
ACM Proceeding of the International Conference

on Computer Graphics, Imaging and Visualization, CGIV'0,

Penang, Malaysia, July 2004, pp. 159-164

more generic available information processing tools. The framework works as an exploration shell for its user.

The following sections of the paper explains polymorphic visual information fusion framework, describe our algorithms for inferring a 3D models, introduce some data classification metrics for the quality of input datasets to be used for fusion visualization and present the example about 3D structural information of liquid crystal based on XPS ( $X$-ray Photoelectron Spectroscopy) data and LSCM (3D Laser Scanning Confocal Microscopy) data about the same area of a piece of liquid crystal film.

\section{Polymorphic Visual Information Fusion (PVIF) Framework}

The basic idea is illustrated in figure 1 , in which we take a real physical-chemical experiment as example. Our polymorphic visual information fusion model generates estimation of 3D structure of objects, based on multiple techniques and allows their systematic comparison and fusion. In this example, the data, collected from XPS and LSCM, might be modified by domain specific filters, as well as geometric transformations (such as rotation, cropping, zooming, tilting and data mapping, etc.). After being filtered, their corresponding 3D models are generated. Next, it allows these 3D models to be visualized as a composite model. It can further generate a fuller model by volume-filling algorithm set and measure the differences by using 3D comparison algorithm set. This process is extensible to additional sensor modalities. Overall the system allows domain specific as well as generic geometric algorithms, filters, and transformation algorithms to be easily infused in the processing pathway leading to the comparative 3D model.

In order to explain our information framework, we only use two abstract techniques A and B. At the same time, we use the following concepts and corresponding notations to facilitate explaining our visualization strategy: (i) $I$ - the total information about the experimental object; (ii) Estimation $I$ - estimation of $I$ from experimental data; (iii) $I^{A}$ - the information generated by technique A; (iv) $I^{B}$ - the information generated by technique $\mathrm{B}$; (v) $F$ - framework for the real structure of experimental object; (vi) $F^{A}$. framework for the structure "seen" through technique A; (vii) $F^{B}$ - framework for the structure "seen" through technique B; (viii) $F^{R}$ - framework for the registration between the intersection of frameworks $\mathrm{A}$ and $\mathrm{B}$, i.e.

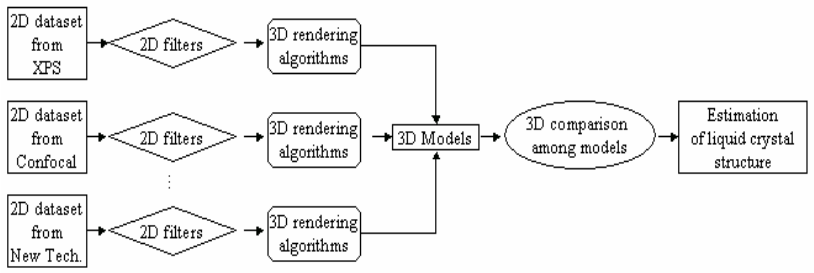

Fig. 1. polysensometric visual information fusion model. In this figure, we derive the 3D models from sets of information, which are generated by different experimental techniques, and compare these models to obtain the structure estimation of our research object to be visualized. The model is extensible

registration framework; (ix) $F^{V}$ - visualization framework for $F^{R}$; (x) Transformations $T[i]$ - any transformation $\mathrm{i}$ applied on information set; (xi) Error $E$ - the difference between two information sets.

In reality, for a complex target of observation we are never able to get the real structure of the target, total $I$ under framework $F$. But we can approximate it as close as possible to obtain estimation $I^{\prime}$. So our objective is to obtain estimation $I$ and visualize it under $F^{V}$, which is close enough to $F$. The relations among these concepts and notations are: the total information, $I$, is corresponding to the total framework, $F ; I^{A}$ is information about $I$ under its framework $F^{A} ; I^{B}$ is information about $I$ under its framework $F^{B} ; F^{A}$ and $F^{B}$ are registered towards $F^{R}$ based on the intersection part between them; $I_{A}{ }^{R}$ is part of information $I^{A}$ after registration under $F^{R} ; I_{B}{ }^{R}$ is part of information $I^{B}$ after registration under $F^{R}$; we use the union of transformed $I^{A}$ and $I^{B}$ as the estimation of I, $I$; $I$ is visualized under $F^{V}$. The inferring algorithm is showed in figure 2 and the specific details will be covered in the next part of this paper.

\section{Algorithms}

The data fusion is achieved through a set of processing: space projection, registration, volumetric filling and comparison, etc. This section interprets those algorithms used to obtain reasonable data fusion results. 
ACM Proceeding of the International Conference

on Computer Graphics, Imaging and Visualization, CGIV'O,

Penang, Malaysia, July 2004, pp. 159-164

\subsection{Sensometric to Geometric Projection Algorithms}

The original input datasets are collected from some sensors. These sensors probably have different space coordinate systems and resolutions. In these cases, we need algorithms to project the sensor domain sample datasets to a common geometric space. After the original datasets processed by these algorithms, they should have the same spatial orientation and resolution and be ready for the following operations. The frequently used transformations are including zoom and tilt etc.

$$
I * T[0] * \ldots * T[\mathrm{n}]->I^{\prime}
$$

In this formula, $I$ is input dataset, $I$ ' is output dataset. $T[\mathrm{i}](0<=\mathrm{i}<=\mathrm{n})$ are $\mathrm{n}+1$ transformations applied on input dataset $I$.

\subsection{Registration Algorithms}

After we project the original sensometric datasets to geometric space, we need the automated registration algorithms to register the voxel in one spatial datasets with the corresponding voxel in the other spatial datasets. In our research, registration algorithms are divided into two groups: 2D registration and $3 \mathrm{D}$ registration. We use mutual information $[7,8]$ or FFT [9] to align our datasets in registration framework, in which the common part of two datasets is normally $2 \mathrm{D}$. Also, we use $3 \mathrm{D}$ registration to fuse two $3 \mathrm{D}$ estimation models, $I_{A}{ }^{V}$ and $I_{B}{ }^{V}$, in visualization framework. Some $3 \mathrm{D}$ transformations are applied on two models, and difference between them is calculated. Difference metrics are discussed later in comparison algorithm. Here least error method is used to decide which transformation will be used to get the final estimation of the real model:

$$
\begin{aligned}
& I_{A}{ }^{V}=f_{i}^{A}\left[I^{A}\right] ; \\
& I_{B}{ }^{V}=f_{i}^{B}\left[I^{B}\right] ; \\
& \mathrm{E}^{\prime}=\operatorname{Diff}\left(I_{A}{ }^{{ }}, I_{B}{ }^{{ }}\right) ;
\end{aligned}
$$

In these formula, $f_{i}$ means ith transformation on dataset $\mathrm{A}$, Diff means difference metric calculation between two parameters.

\subsection{Comparison Algorithms}

Comparison algorithms are used for inferring the final 3D model. It checks if the loop ending conditions have been met. There are many 2D whole-image level comparison algorithms, such as mean-absolute-error (MAE), root-mean-square-error (RMSE), peak signal-tonoise ratio (SNR) etc. $[1,2]$. In this example system, we used comparison algorithms that are extension of those used in 2D. The greater MAE and RMSE, the more different these two 3D models are; SNR is on the contrary; the closer $\mathrm{p}$ is to 1 , the more correlated are the two sample images.

\subsection{D Model Inferring Algorithm}

We use 3D model inferring algorithm to infer the final 3D visualization model for the object of interest (see figure 2). The algorithm is composed of two loops. We explain the algorithm by using two techniques, A and B, as an example. First, the input datasets from sensors generate 3D models of their own by using hypotheses $\mathrm{H}_{\mathrm{a}}$ and $\mathrm{H}_{\mathrm{b}}$ respectively. Then, these two $3 \mathrm{D}$ models are projected onto registration framework, $F^{R}$. In registration framework, the intersection parts of $F^{A}$ and $F^{B}$ are registered by applying a series of transformations. Least errors are used to get the optimum registration position. After registered, $I_{A}$ and $I_{B}$ are compared to verify the hypotheses at the very beginning. Least error principle is also used as the optimum means to obtain the best theoretic 3D model about the object of interest. At last, we use the union of $I_{A}$ and $I_{B}$ from the hypotheses with least error as the estimation of total information $I$.

\section{Data Classification}

Our PVIF framework takes advantage of the intersection parts among datasets extracted from multiple sensors. So it is important to know how much intersection there are in the provided datasets quantitatively. We 
ACM Proceeding of the International Conference

on Computer Graphics, Imaging and Visualization, CGIV'0, Penang, Malaysia, July 2004, pp. 159-164

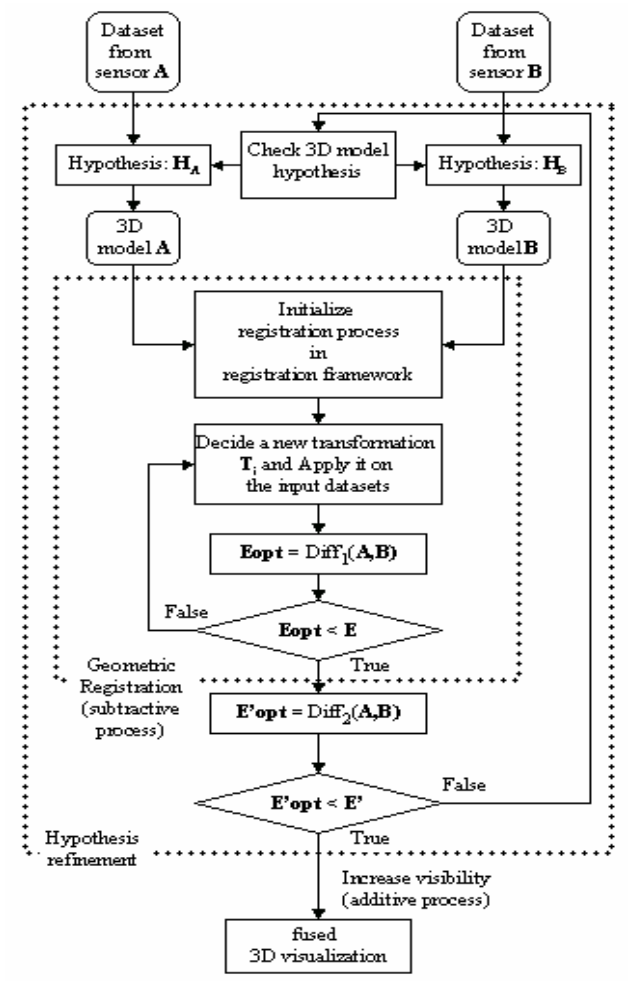

Fig. 2. "pounding-and-inferring" algorithm for 3D model based on sets of information from two techniques $A$ and $B$

propose several metrics, such as intersection degree, exposition ratio and inferring confidence, etc., to tell the quality of our data for fusion visualization. In the following section, I will use an example to explain how these metrics are to be used.

For example, if we have a sample datasets which contains 2 sets of data from every instrument among LSCM, XPS and AFM. An external reference is used to locate the shooting area for all datasets in an experiment. The spatial relationship among these datasets is illustrated in figure 3 .

\subsection{Intersection degree}

We describe intersection degree as percentages of the zones on the surface, which are covered by one dataset, two datasets, ..., and all datasets in sample, over the total surface area shot by all instruments. From spatial relationship illustrated in figure 3 , we know some pixels on the surface of object of interest are located in one dataset; some are in two datasets, etc. We call it degree of pixel. If only one dataset owns a certain pixel, then its degree is 1 ; two datasets own it, then its degree is 2 . The biggest degree value for any pixel is the number of datasets. We can count the degree of all pixels of our datasets. After this, we can obtain the pixel number with each different degree value in the datasets. Then the percentages of each degree are calculated by the pixel number of each degree over the total pixel number. The sample result are illustrated one the bottom of figure 3 .
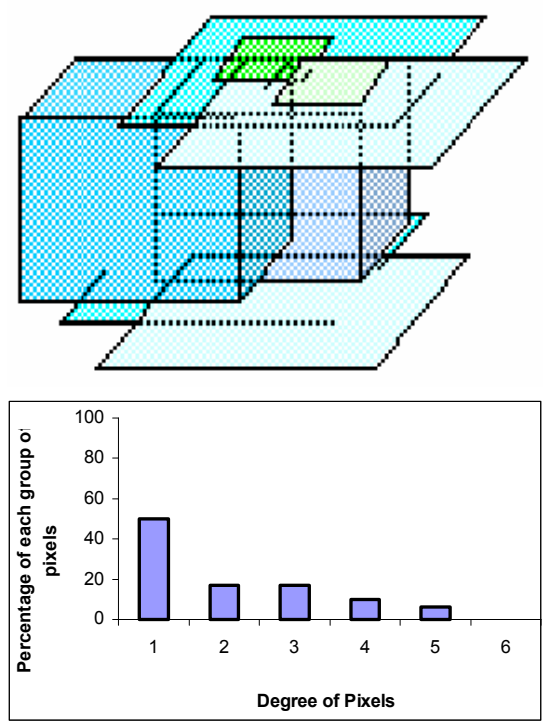

Fig. 3. top: The spatial locations of all datasets in a liquid crystal experiment. Cyan color: XPS data; Blue: LSCM data; Green: AFM data. bottom: intersection degree.

\subsection{Exposition ratio and inferring confidence}

Exposition ratio (ER) is defined as the ratio of the union of a certain number of datasets to the sum of these datasets, while inferring confidence (IC) is the ratio of the intersection a certain number of datasets to the sum of these datasets.

$$
\begin{aligned}
& \mathrm{ER}=\left(\mathrm{I}_{1} \mathrm{u} \mathrm{I} \mathrm{I}_{2} \mathrm{u} \ldots \mathrm{u} \mathrm{I} \mathrm{I}_{\mathrm{m}}\right) /\left(\mathrm{I}_{1}+\mathrm{I}_{2}+\ldots+\mathrm{I}_{\mathrm{m}}\right) ; \\
& \mathrm{IC}=\left(\mathrm{I}_{1} \mathrm{n} \mathrm{I}_{2} \mathrm{n} \ldots \mathrm{n} \mathrm{I}_{\mathrm{m}}\right) /\left(\mathrm{I}_{1}+\mathrm{I}_{2}+\ldots+\mathrm{I}_{\mathrm{m}}\right) ;
\end{aligned}
$$


ACM Proceeding of the International Conference

on Computer Graphics, Imaging and Visualization, CGIV'0,

Penang, Malaysia, July 2004, pp. 159-164

Before we can calculate exposition ratio and inferring confidence using formula 5 and 6 , there is one problem. It is how we are going to calculate the information quantity in a dataset. Our solution is to use the total shooting area as the information in a dataset:

$$
I=\sum_{i} \text { Area }_{i}
$$

For example, the shooting area is $123 \times 185$ microns and there are 35 images in our LSCM dataset. So the information in the dataset should be $123 * 185 * 35$, i.e. $7.96 \mathrm{e}+5$.

So it is easy to calculate exposition ratio and inferring confidence using our definitions and our dataset information in table 2. For example, if we want to calculate ER and IC between XPS1 and XPS2. First we obtain the sum of $\mathrm{I}_{\mathrm{xps} 1}$ and $\mathrm{I}_{\mathrm{xps} 2}$. It is $2 *(2 *(200 * 200))$, i.e. 1.6e +5 . Then intersection information of $\mathrm{I}_{\mathrm{xps} 1}$ and $\mathrm{I}_{\mathrm{xps} 2}$ is $2 * 9,000$, i.e. $1.8 \mathrm{e}+4$. Therefore the union information of these two datasets is the difference between $1.6 \mathrm{e}+5$ and $1.8 \mathrm{e}+4$, i.e. $1.42 \mathrm{e}+5$.

\section{System Architecture and Working Process}

The architecture of our shell system is illustrated in figure 4. The shell is composed of such components as task controller, flowchart object store and some other functional parts, such as flowchart designer, data combiner, information verifier etc. Task controller works as a central control manager. It controls the state of our program and coordinates the work of the functional parts. Its work includes deletion of a flowchart object from the flowchart store, let the flowchart designer insert a flowchart object into the store according to the requirement of the user, injection of input data through the data combiner, declaration and definition of filters and algorithms through filter/algorithm combiner, verification of information in the flowchart object through verifer and driving the transformation engine to process the data following the controls in flowchart object and generating the final model. The flowchart object store is used to store all the information about data and operations what will be applied on the data. Information transformation engine drives the input data through the control of the flowchart and generate 3D results. Also, hypothesis refinement can be done by this component.

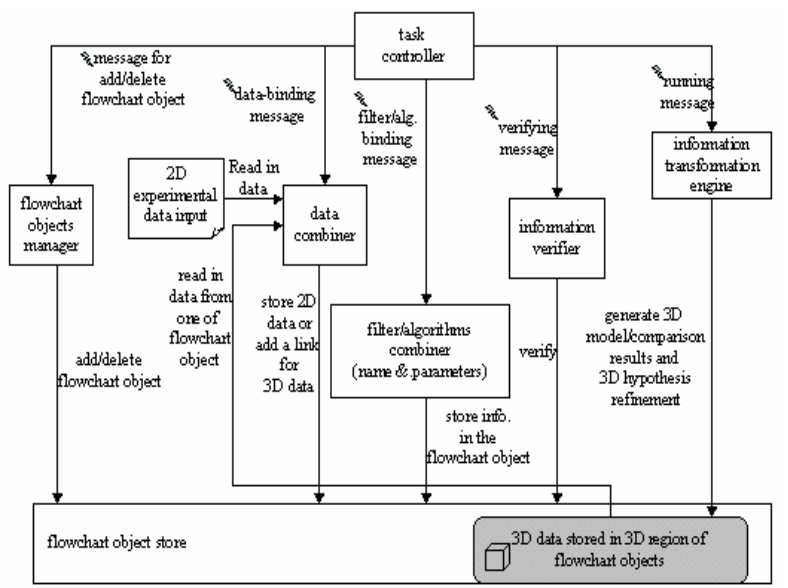

Fig. 4. System architecture

There are total 5 phases to run the whole process: design phase, name-binding phase, parameter-binding phase, verification phase and run phase. During design phase, a flowchart object can be designed by using flowchart designer. There are two kinds of flowchart object in the project: one is to take $2 \mathrm{D}$ experimental data as input and generate a 3D model; another is to take 3D models, which are generated by some flowchart object before, as input and generate the fusion 3D object or comparison between two 3D models. During name Binding Phase, we declare filters and algorithms. Another important thing in this phase is to bind experiment method and image data. During parameter Binding Phase, we set parameters for each filter and algorithm declared in the current flowchart object. During verification Phase, all those input information need to be verified before the data are to be transformed following the datapath defined by the flowchart object. During run Phase, a new 3D model will be created from 2D or 3D data. This model will be displayed in a separate window for further visualization. In this phase, user can also make parameters of filters and algorithms change on the fly in order to get a content result. These changes will be flush back to the flowchart aggregation component when the user pushes a button in the 3D model presentation window.

\section{Case Study}

We visualize the fused information about liquid crystal polymeric film, which was collected by using XPS and 
ACM Proceeding of the International Conference

on Computer Graphics, Imaging and Visualization, CGIV'0,

Penang, Malaysia, July 2004, pp. 159-164

LSCM [5,6]. The polymeric film is 2 microns thick and composed of two non-equally distributed polymers: PVC and PMMA. In XPS, only top and bottom surface with 0.01 microns thick into film have contribution to top image and bottom image of 265 by 265 pixels respectively. The dimension of the shot area is 700 by 700 microns. We also know that the highest pixel intensity in the image corresponds to 35 percent PVC and the lowest pixel intensity is to 5 percent PVC. LSCM generates 32 images of 600 by 800 pixels in this example. The shot area is 300 by 400 microns. The first and last LSCM images are in the depth 0.2 microns to the surface of the film. The highest pixel intensity in the fourth image corresponds to 70 percent PVC and the lowest pixel intensity of the same image is to 20 percent PVC. Also, we are ensured that the shot area in XPS is partially overlapped with that in LSCM. Now our task is to visualize the fusion information based on data from XPS and LSCM. Also, we let user choose theoretic model to fill space in between XPS top and bottom images and then compare them with fusion model. Thus, user can obtain a proposed theoretic model, which is closest to experimental data.

Our system is used to process the input XPS and LSCM datasets. First, we design two flowchart objects to process XPS and LSCM datasets respectively. These two flowchart objects should adjust the resolution, image dimension, concentration mapping, etc., in order to let them match for fusion. After 3D models of each sensor domain are generated, we fused them to form a combined 3D model. And we also use 3D in-filling algorithms to fill the space between XPS images to form several theoretic 3D models. Then comparison metrics are calculated and the best proposed 3D structure of material is decided.

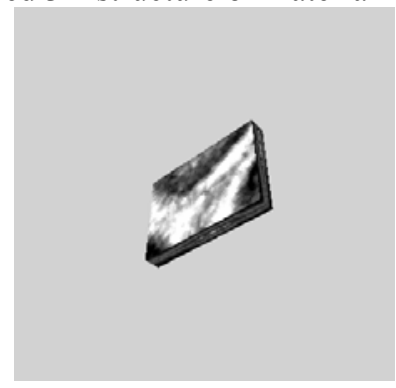

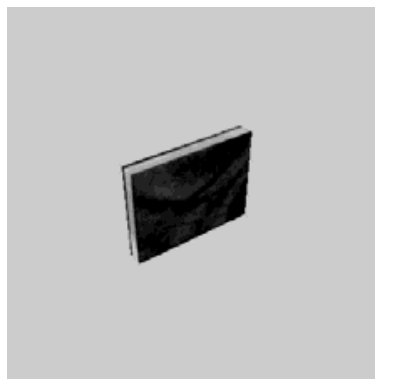

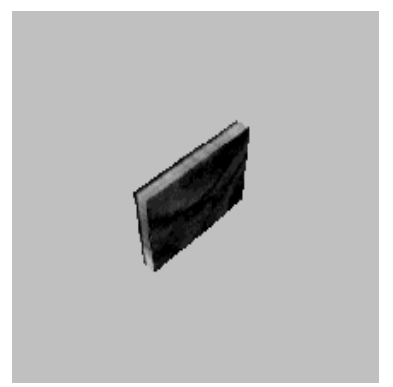

Fig. 5. Result images. Top: the stack of result images by combining XPS data and LSCM data in 3D presentation. We put the top image of XPS on the top of $1^{\text {st }}$ image of LSCM and the bottom image of XPS under the last image of LSCM. Middle: the difference between theoretic model 1 and fusion model. MAE: 119.3, SNR: 129.8 . Bottom: the difference between theoretic model 2 and fusion model. MAE $=87.3 ;$ SNR $=99.0$

The result of our example is illustrated in the attached figures. In figure 5, a fused 3D model of XPS and LSCM is illustrated. We put XPS top image on the top of the $1^{\text {st }}$ image of LSCM image stack and XPS bottom image at the bottom of the last image of LSCM image stack to form a composite 3D model based on these two techniques. Also, we use linear insertion algorithm insert 30 images between top image and bottom image of XPS datasets. Thus both datasets have the same number of images. After using all filters, they also have the same resolution, dimension and been well registered. So we use our comparison metrics, MAE and SNR, to compare these two 3D models. The results are: $\mathrm{MAE}=119.3 ; \mathrm{SNR}=129.8$. The comparison results are illustrated in figure 5 . We also use another different formula to fill the space in between the top and bottom image: $2 *$ topPixel* ${ }^{*}+$ botPixel $^{*}(1-\mathrm{x})$. Comparison between the new 3D XPS model and LSCM model is 
ACM Proceeding of the International Conference

on Computer Graphics, Imaging and Visualization, CGIV'0,

Penang, Malaysia, July 2004, pp. 159-164

calculated: $\mathrm{MAE}=87.3 ; \mathrm{SNR}=99.0$. So according to MAE and SNR, the second in-filling function generates a result closer to LSCM. The results are illustrated in figure 5.

From the above case study, we see our system can generate a fused and theoretic 3D model based on a set of experimental data and user-defined algorithms. The user might propose different theoretic 3D models and compare these theoretic models with real experimental data. Thus the best theoretic model of chemical structure of the material of interest could be chosen from the proposals based on the comparison metrics.

\section{Conclusion}

In this paper, we propose Polymorphic Visual Information Fusion (PVIF) Model to infer estimation of total information of the object of interest based on partial information from multiple instruments, and implement an integrated system for automating to fuse the partial information and compare different 3D models to refine the best operations for a certain dataset and testify the validity of a theoretic 3D model. Our system can be used as a tool for chemists and physicists to explore the structure of a material.

There are still a lot of interesting and complicated issues which would need more attention. Among these difficulties, 2D/3D registration algorithm itself is a challenge in image processing; how to find a good 3D comparison metric is also interesting; how to describe a proposed 3D model is not an easy job. Rendering speed problem is also important in the fields of image processing and graphics. The work has been supported by National Science Foundation (NSF) Grant NSF-0113724.

\section{References}

[1] .Kwansik Kim, Alex Pang, Ray-based Data Level Comparisons of Direct Volume Rendering Algorithms. Technical
Report UCSC-CRL-97-15, UCSC Computer Science Department, 1997.

[2] Rafael Gonzalez and Richard Woods, "chapter 8, image compression", Digital Image Processing, 2nd edition, prentice Hall, 2001, pp419-420.

[3] Schiers C, Tiede U, Hohne KH 1989, Interactive 3Dregistration of image volumes from different sources. In: Lemke HU et al. (eds), Computer Assisted Radiology (Proc. CAR '89), Springer, Berlin.

[4] Hohne, K.H., Bomans, M, et al, 3D-Visualization of tomographic volume data using the generalized voxel-model, $\mathrm{CH}$ Volume Visualization workshop.

[5] Laurie A. Broadwater, "The correalation of X-Ray Photoelectron Spectroscopy And Laser Scanning Confocal Microscopy”, thesis, Kent State University, May, 2003.

[6] Kateryna Artyushkova, "Application of Multitechnique Correlation And Multivariate Analysis To Heterogeneous Polymer Systems", dissertation, Kent State University, Dec., 2001.

[7] William M. Wells, Paul Viola, Hideki Atsumi and Shin Nakajima, Multi-Modal Volume Registration by Maximization of Mutual Information, Medical Image Analysis, vol. 1, no. 1, pp. 35--51, March 1996.

[8] Michael E. Leventon and Eric L. Grimson, Multi-Modal Volume Registration Using Joint Intensity Distributions, in Medical Image Computing and Computer-Assisted Intervention MICCAI'98, ed. by W.M. Wells, A. Colchester, S. Delp, Cambridge, MA, Springer LNCS 1496, pp 1057.

[9] P.E. Anuta, Spatial registration of multispectral and multitemporal digital imagery using fast fourier transform techniques. IEEE Trans. on Geoscience Electronics, GE8(4):353--368, October 1970. 